\title{
Emplois de l'aDcp en rivière : une revue de synthèse
}

\author{
Uses of aDcp in rivers : a review \\ JÉRÔME LE COZ, BERNARD CHASTAN, FABIEN VEDIE, GUILLAUME DRAMAIS \\ Cemagref, Unité de Recherche Hydrologie-Hydraulique, \\ 3 bis quai Chauveau CP 220 F-69336 Lyon cedex 09, France
}

$F^{\prime}$ or the last fifteen years, acoustic Doppler current profilers (aDcp) have been increasingly used to measure river discharge. Originally designed for oceanographical applications, aDcp can provide vertical profiles of $3 D$ instantaneous flow velocity, referenced in space and time at a sampling rate of up to about $5 \mathrm{~Hz}$. ADcps emit ultrasonic waves whose rated frequency typically ranges from 300 to 3,000 $\mathrm{kHz}$. Water velocity is measured by Doppler effect, so that no local parameter calibration is required. Due to some technical restrictions it is impossible to measure velocity in certain parts of the river cross-section. Uncertainty assessment is made difficult by the complexity of the measurement process and potential errors that stem from field deployment. Consequently practitioners are looking for guidelines and corrective methods in order to assess the quality of discharge measurement, especially in case of moving bottom or complex flows (eddies, recirculating flows, secondary currents, etc.). Besides, today much experimental effort is dedicated to making the most of this versatile and very slightly intrusive device: bed topography, velocity field measurements, estimate of bed load velocity or suspended solid fluxes. This variety of data can be obtained rapidly and economically even in tough field conditions. Therefore Doppler profilers appear as promising tools for the study of river systems.

\section{INTRODUCTION}

L'acoustique sous-marine a connu de grandes avancées scientifiques et techniques en raison de l'importance stratégique qu'ont pris les sous-marins dès la $1^{\text {re }}$ Guerre mondiale. Ainsi, les profileurs acoustiques de vitesse à effet Doppler (aDcp, pour ang. acoustic Doppler current profiler) ont été initialement développés pour l'exploration dans le temps et dans l'espace de profils verticaux de vitesse dans les domaines océaniques et côtiers. Des adaptations technologiques dans les années 1990 ont permis d'étendre le champ d'application des aDcp aux eaux continentales moins profondes [Yorke et al. 2002].

A l'heure actuelle, les mesures par aDcp sont de plus en plus pratiquées, notamment par les organismes français ayant en charge le suivi du débit des rivières (DIREN, CNR, EDF, IRD, VNF,...). Les équipes d'hydrométrie sont en effet séduites par le gain de temps de mise en œuvre et la possibilité de réaliser des jaugeages dans des conditions pour lesquelles les techniques conventionnelles sont très lourdes voire impraticables. Parallèlement à cette utilisation opérationnelle, de nombreux efforts de recherche portent actuellement sur l'exploitation de l'ensemble des données qu'offre potentiellement l'aDcp : bathymétrie, champ de vitesse 3D, flux de sédiments transportés en suspension et par charriage.

Parmi tous les appareils à ultrasons réalisant des mesures de vitesse d'écoulement par effet Doppler et utilisables sur les cours d'eau, il convient de distinguer d'emblée les vélocimètres Doppler ou $\mathrm{aDv}$ (acoustic Doppler velocimeter, mesure de vitesse ponctuelle), les débitmètres Doppler (mesure d'une vitesse moyenne dans le faisceau acoustique) et les profileurs Doppler $(\mathrm{aDcp}$, mesure de vitesses réparties le long d'un axe). Cet article propose une présentation synthétique de ce dernier type d'appareil, ainsi qu'un tour d'horizon de la diversité de ses emplois en rivière, à la lumière de la littérature scientifique internationale et de retours d'expérience de la part des utilisateurs.

N.B. On emploie dans tout ce texte l'acronyme aDcp pour désigner tout type de profileur acoustique de vitesse à effet Doppler, sans distinction de modèle ni de constructeur. En effet, comme pour mobylette ou frigidaire, l'usage courant a retenu ce terme générique issu par antonomase d'un nom de modèle commercial (ADCP $\left.{ }^{\mathrm{TM}}\right)$. Certains matériels commerciaux courants (profileurs, accessoires et logiciels) sont cités ou décrits succinctement à titre illustratif, sans prétention à l'exhaustivité ni jugement comparatif.

\section{II — PRÉSENTATION GÉNÉRALE DE L'ADCP}

\section{II.1 PRINCIPE DE LA VÉLOCIMÉTRIE PAR EFFET DOPPLER}
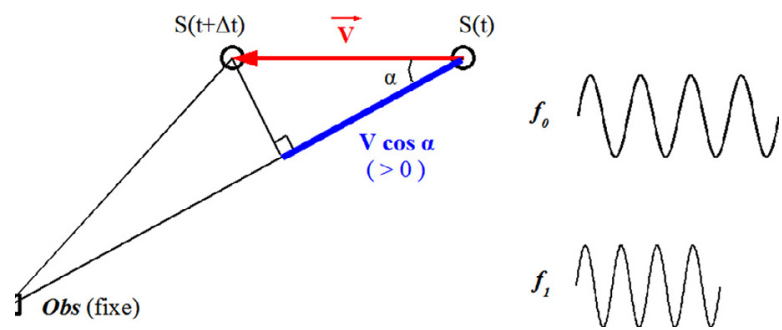

Figure II-1 : l'effet Doppler 
La mesure de la vitesse en un point de l'écoulement tire parti de l'effet Doppler, énoncé par le physicien autrichien Christian Doppler [Doppler 1842] : un observateur Obs fixe (dans son propre référentiel) perçoit un décalage de fréquence $\Delta f$ d'une onde (ici sonore) émise par une source $S$ mobile de vitesse $\vec{V}$ dans le référentiel lié à l'observateur (cf. figure II-1). Le son parvient à l'observateur plus aigu si la source se rapproche de lui, et plus grave si elle s'en éloigne. Le décalage fréquentiel $\Delta f$ est proportionnel à la fréquence d'émission $f_{0}$ et à la vitesse radiale de la source $V \cos \alpha$ :

$$
V \cos \alpha=C \frac{\Delta f}{f_{0}} \text { avec } \Delta f=f_{1}-f_{0}
$$

$f_{1}$ étant la fréquence du signal reçu par l'observateur, $f_{0}$ la fréquence du signal émis par la source et $C$ la célérité de l'onde dans le milieu.

Dans le cas d'un aDcp, un signal ultrasonique est émis dans l'eau par une céramique piézo-électrique, rétro-diffusé par les particules en suspension - supposées de même vitesse que l'écoulement, puis reçu par la même céramique. L'effet Doppler se produit donc deux fois, la première fois de la céramique à la particule, la seconde fois de la particule à la céramique. Comme $\left|f_{1}-f_{0}\right| \ll f_{0} \quad($ car $V<<C)$, le décalage Doppler est simplement doublé :

$$
V \cos \alpha=\frac{C}{2} \frac{\Delta f}{f_{0}} \text { avec } \Delta f=f_{2}-f_{0}
$$

$f_{0}, f_{1}, f_{2}$ étant respectivement les fréquences du signal émis par l'appareil, reçu et ré-émis par les particules et finalement revenu à l'appareil.

Ce principe de mesure peut être appliqué à une émission continue (cas des débitmètres Doppler continus [Birgand et al. 2005]) ou à l'émission d'impulsions (Doppler pulsé). Seul le Doppler pulsé permet d'obtenir des profils de vitesse (cf. $\S$ II.2). Lorsque le décalage fréquentiel est analysé selon les principes exposés ci-dessus, on parle de Doppler «à impulsions incohérentes » ou narrowband: pour chaque impulsion (relativement longue en général) émise, la fréquence de l'écho est analysée.

Dans le cas de mesures "à impulsions cohérentes 》 (pulse-coherent), ce n'est plus ce décalage de fréquence qui est exploité mais, de façon équivalente, le déphasage entre les échos de deux impulsions rapprochées [RDI 1996]. A l'émission, ces deux impulsions sont de même forme et de même phase, la deuxième n'étant émise que lorsque l'écho de la première a été entièrement reçu. La différence de phase entre les deux échos étant connue modulo $2 \pi$, il est nécessaire de définir a priori une intensité de vitesse maximale mesurable en-deçà de laquelle le déphasage sera inférieur à $\pi$ et l'interprétation univoque. Le délai fixé entre deux impulsions successives réalise ainsi un compromis entre la gamme de vitesses mesurables sans ambiguïté et la portée [Lemmin et al. 1997, SonTek 2001].

Une troisième technologie dite broadband permet de s'affranchir de cette contrainte en remplaçant chaque impulsion longue par une série d'impulsions courtes codées en phase (succession semi-aléatoire de 0 et $\pi$ ) : le bruit engendré par la double exposition des particules (les deux impulsions sont présentes simultanément dans la colonne d'eau) est résorbé par la moyenne permise par le grand nombre d'impulsions courtes [Simpson 2001]. Grâce à une méthode d'autocorrélation, une trop grande distorsion des signaux rétro-diffusés permet d'écarter les mesures corrompues par des obstacles (végétation, débris, poissons, bulles, etc.).

Les technologies broadband et a fortiori pulse-coherent permettent de réduire la dispersion des mesures (ou «bruit» Doppler) et d'atteindre une meilleure résolution spatiale. En revanche, la portée est plus réduite, à fréquence de signal donnée, que pour la technologie narrowband, plus robuste et à cadence de mesure plus élevée.

\section{II.2 CONFIGURATION TECHNIQUE DE L'ADCP ET ÉCHANTILLONNAGE}

Seule la vitesse radiale $V \cos \alpha$ (selon l'axe du faisceau) peut être mesurée par effet Doppler. Au moins trois faisceaux non coplanaires sont donc nécessaires pour reconstituer les trois composantes de la vitesse, à partir de relations trigonométriques simples. Les constructeurs rajoutent parfois un quatrième faisceau permettant de définir un facteur de qualité de la mesure (cf. § III.3). Les transducteurs (céramiques émettrices/réceptrices) se présentent en général organisés symétriquement en plan, avec un angle $\beta$ constant par rapport à la verticale (voir figure II-2). Quasiment tous les profileurs commerciaux respectent cette configuration particulière (profileurs dits monostatiques). Le diamètre des céramiques varie typiquement de 2 à $30 \mathrm{~cm}$ en fonction des modèles.

Selon chaque faisceau, pour chaque séquence d'écho analysée, la profondeur des particules rétrodiffusantes est déterminée grâce au temps de retour du signal émis et à la célérité $C$ du son. Cette dernière est recalculée en fonction de la température de l'eau mesurée au niveau de l'appareil (la salinité est en pratique supposée nulle en rivière). Le découpage temporel du signal reçu (« range gating ») permet ainsi d'établir un profil vertical des vitesses. Pour chaque segment vertical, une moyenne pondérée des vitesses est affectée au centre du segment ou cellule («bin») de hauteur définie par l'opérateur (de 10 à $100 \mathrm{~cm}$ typiquement, selon la fréquence des ultrasons et le mode de mesure). En fonction du diamètre de la céramique, de la hauteur des cellules et de l'ouverture du faisceau acoustique (cf. § III.2), le volume d'échantillonnage peut mesurer typiquement de quelques $\mathrm{cm}^{3}$ à plus de $1000 \mathrm{~cm}^{3}$ [Muste et al. 2004c].
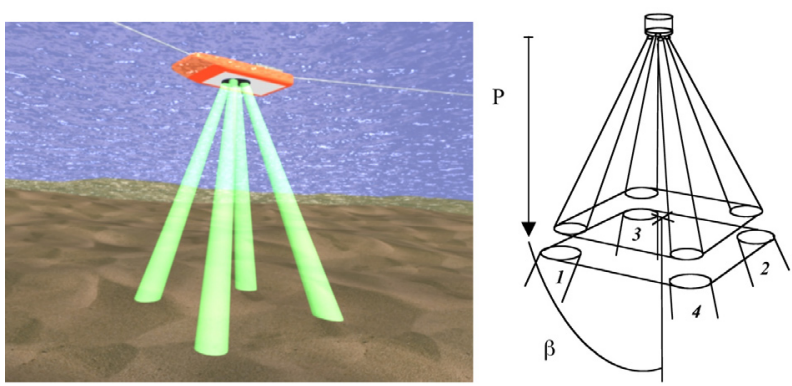

Figure II-2 : configuration Janus d'un aDcp monostatique à quatre faisceaux 
Contrairement à un courantomètre classique ou même un vélocimètre Doppler $(\mathrm{aDv}), \mathrm{l}$ 'aDcp n'effectue donc pas une mesure ponctuelle de la vitesse, mais une reconstruction de cette vitesse à partir de mesures unidirectionnelles sur trois ou quatre volumes de mesure à une profondeur donnée, selon l'axe de chaque faisceau. D'une part, cette reconstruction n'est valide que si le champ de vitesse est suffisamment homogène dans la couche horizontale considérée (cf. § III.3). D'autre part, la distance entre volumes d'échantillonnage augmente rapidement proportionnellement à la profondeur : dans le cas décrit figure II-2, ces volumes élémentaires occupent les coins d'un carré de côté $\sqrt{2} P \tan (\beta)$ soit environ la moitié de la profondeur $P$ pour un écartement $\beta=20^{\circ}$. En fonction de la cadence d'échantillonnage (jusqu'à 3 ou $5 \mathrm{~Hz}$ maximum typiquement) et de la vitesse de déplacement de l'aDcp, on aboutit facilement à des recouvrements de volumes mesurés plusieurs fois successivement. Le jeu de vitesses obtenu est alors le résultat de moyennes spatiales et temporelles complexes.

En rivière, l'aDcp est généralement utilisé monté sur un support flottant mobile, même s'il peut être fixé au fond, sur une bouée, voire sur le côté d'une section pour suivre en continu les vitesses le long d'une horizontale [Wang et al. 2005]. Un tel aDcp transversal (ou horizontal) à deux faisceaux horizontaux vient ainsi d'être installé par la CNR sur la Saône à Lyon. Les problèmes méthodologiques et métrologiques ne sont pas les mêmes selon que le profileur est mobile [Muste et al. 2004c] ou fixe [Muste et al. 2004b], ne serait-ce que parce que la vitesse d'écoulement, mesurée dans le référentiel de l'aDcp, doit alors être rapportée au référentiel terrestre. Tangage et roulis notamment peuvent être compensés dans une certaine mesure si le support de l'aDcp est muni de capteurs d'inclinaison. Dans le cas contraire, il faut s'assurer que le capteur demeure le plus horizontal et stable possible.

\section{II.3 CARACTÉRISTIQUES DES PROFILEURS DOPPLER ACTUELS}

Certaines équipes de recherche développent leurs propres outils hydroacoustiques, tel l'ADVP [Lemmin et al. 1997] (Acoustic Doppler Velocity Profiler, $1 \mathrm{MHz}$, pulse-coherent), notamment pour l'étude fine des écoulements turbulents (cf. $\S$ V.3) en laboratoire et même sur des cours d'eau à très faible tirant d'eau [Franca 2005]. L'ADVP est un profileur dit bistatique : un seul faisceau vertical est émis par une céramique centrale, les échos étant reçus par plusieurs récepteurs périphériques inclinés.

A l'heure actuelle toutefois, la plupart des profileurs Doppler du marché (monostatiques) sont commercialisés par deux constructeurs américains: Teledyne RDI (www. rdinstruments.com) propose une gamme d' $A D C P^{1}$ (Acoustic Doppler Current Profiler, broadband, modes 5 et 11 à impulsions cohérentes), dont un modèle StreamPro dédié aux faibles tirants d'eau. SonTek/YSI (www.sontek.com) développe et commercialise 1' $A D P^{2}$ (Acoustic Doppler Profiler, narrowband) et ses variantes mini-ADP (taille réduite pour faibles tirants d'eau) et $P C$-ADP (pulse-coherent, sans suivi de fond, $\beta=15^{\circ}$ ).

Enfin, un montage intégré sur mini-catamaran du profileur norvégien Aquadopp ${ }^{3}$ de NorTek (Qliner commercialisé par Qmetrix, http ://www.qmetrix.com) est également adapté au jaugeage des cours d'eau modestes (largeur 1 à $30 \mathrm{~m}$, profondeur 0,3 à $5 \mathrm{~m}$ ). Tous les faisceaux sont émis dans le plan vertical aligné sur l'axe longitudinal de l'embarcation, supposée elle-même alignée sur l'écoulement moyen. La mesure du profil de vitesse dans ce plan est assurée par deux fais-

1. ADCP est une marque déposée par Teledyne RDI

2. ADP est une marque déposée par SonTek /YSI.

3. Aquadopp est une marque déposée par NorTek/AS.

Tableau II-1 : caractéristiques des aDcp commerciaux les plus courants

\begin{tabular}{|c|c|c|c|c|c|}
\hline Modèle & $\begin{array}{l}\text { Fréquence des } \\
\text { ultrasons }(\mathrm{kHz})\end{array}$ & $\begin{array}{c}\text { Profondeur } \\
\text { maximale (m) }\end{array}$ & $\begin{array}{c}\text { Nombre } \\
\text { et configuration } \\
\text { des faisceaux }\end{array}$ & $\begin{array}{c}\text { Compas } \\
\text { et captures } \\
\text { d'inclinaison }\end{array}$ & Suivi de fond \\
\hline $\begin{array}{c}\text { T-RDI Workhorse } \\
\text { RioGrande }\end{array}$ & $\begin{array}{c}600 \\
1200\end{array}$ & $\begin{array}{l}100 \\
26\end{array}$ & 4 , conf. Janus $\left(\beta=20^{\circ}\right)$ & oui & oui \\
\hline $\begin{array}{c}\text { T-RDI } \\
\text { StreamPro }\end{array}$ & 2400 & $2-4$ & 4 , conf. Janus $\left(\beta=20^{\circ}\right)$ & non & oui \\
\hline \multirow{4}{*}{$\begin{array}{c}\text { SonTek } \\
A D P\end{array}$} & 500 & $70-120$ & \multirow{4}{*}{$\begin{array}{c}3\left(\beta=25^{\circ}\right), \text { options à } 2 \\
\text { ou } 4 \text { faisceaux }\end{array}$} & \multirow{4}{*}{$\begin{array}{c}\text { oui (cas du } \\
\text { RiverSurveyor) }\end{array}$} & \multirow{4}{*}{$\begin{array}{l}\text { oui (sauf en pluse- } \\
\text { coherent) }\end{array}$} \\
\hline & 1000 & $25-40$ & & & \\
\hline & 1500 & $15-25$ & & & \\
\hline & 3000 & $3-6$ & & & \\
\hline \multirow{2}{*}{$\begin{array}{c}\text { SonTek } \\
\text { mini-ADP }\end{array}$} & 1500 & 25 & \multirow{2}{*}{3 faisceaux $\left(\beta=25^{\circ}\right)$} & \multirow{2}{*}{ oui } & \multirow{2}{*}{ idem } \\
\hline & 3000 & 6 & & & \\
\hline $\begin{array}{l}\text { NorTek } \\
\text { QLiner }\end{array}$ & $\begin{array}{l}1000 \\
2000\end{array}$ & - & $\begin{array}{l}2 \text { faisceaux }\left(\beta_{1,2}=25^{\circ}\right) \\
+1 \text { faisceau }\left(\beta_{3}=20^{\circ}\right)\end{array}$ & oui & $\begin{array}{l}\text { non (mesures fixes } \\
\text { uniquement) }\end{array}$ \\
\hline
\end{tabular}


ceaux à $+25^{\circ}$ et $-25^{\circ}$ de la verticale et un troisième faisceau pointant vers l'avant, à $20^{\circ}$ sous l'horizontale, de manière à réduire la zone aveugle. Un quatrième faisceau, pointant à la verticale, fonctionne en échosondeur pour donner la profondeur au droit du système. Un système très similaire appelé BoogieDopp est développé par NorTek/USA [Cheng et al. 2003].

Les caractéristiques des modèles commerciaux les plus répandus sont résumées dans le Tableau II-1, à partir des informations fournies par les constructeurs.

\section{III — PRINCIPALES CONTRAINTES TECHNIQUES}

\section{III.1 ZONE AVEUGLE ET RÉSONANCE DE L'ÉMETTEUR}

L'aDcp ne peut pas mesurer de vitesses à proximité de la surface de l'écoulement sur une hauteur correspondant à la profondeur d'immersion des céramiques $(5$ à $100 \mathrm{~cm}$ en pratique). En outre, il existe un délai minimum indispensable entre l'émission et la réception pour que la résonance de la céramique ne vienne pas brouiller l'enregistrement de l'écho. Ce délai, fonction de la fréquence des ultrasons, se traduit par une distance supplémentaire avant la première cellule de mesure (cf. figure III-1). Jusqu'à ces dernières années, cette distance perdue était typiquement de $6 \mathrm{~m}$ pour un appareil à $75 \mathrm{kHz}$, contre environ $50 \mathrm{~cm}$ à $1200 \mathrm{kHz}$ [RDI 1996]. Depuis, afin de s'adapter au jaugeage à très faibles tirants d'eau, les constructeurs ont amélioré les performances des céramiques, réduisant la zone aveugle à $5 \mathrm{~cm}$ pour un aDcp $1200 \mathrm{kHz}$. Cependant, l'intrusion dans l'écoulement du châssis pseudo-sphérique portant les céramiques induit une déformation locale des lignes de courant [Gartner et al. 2002]. La sous-estimation de la composante radiale de la vitesse qui en résulte limite physiquement la mesure à proximité immédiate du capteur, d'autant plus que l'écoulement apparent est rapide.

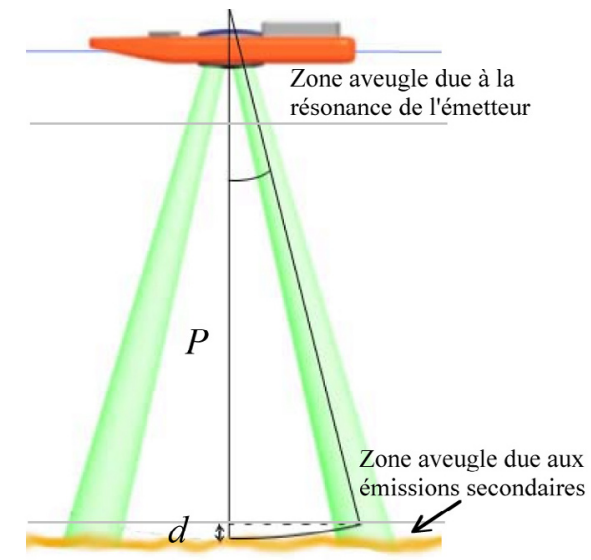

Figure III-1 : tranches d'eau que l'aDcp ne peut pas explorer

\section{III.2 INTERFÉRENCE DES ÉMISSIONS SECONDAIRES}

Chaque céramique piézoélectrique émet un faisceau d'ultrasons concentrés autour d'une direction principale. L'ouverture du lobe acoustique principal (i.e. la largeur du faisceau à $-3 \mathrm{~dB}$ ) décroît avec la fréquence de l'aDcp. Elle vaut environ 1,5 pour un ADCP Teledyne RDI Broadband à $1200 \mathrm{kHz}$ [RDI 1996] ou pour un ADP SonTek à 1500 $\mathrm{kHz}$ [Rennie et al. 2002]. Ceci correspond typiquement à un doublement du diamètre du faisceau à $2 \mathrm{~m}$ de la céramique. Cependant des émissions secondaires («side-lobes ») extérieures à ce cône peuvent rencontrer le fond de la rivière et alors engendrer un écho d'intensité comparable à la rétrodiffusion du faisceau principal par les particules en suspension. Cet écho parasite perturbe la mesure de vitesse, en revenant à l'émetteur. Les premiers échos parasites enregistrés sont potentiellement ceux qui ont parcouru le chemin le plus court, soit deux fois le tirant d'eau $P$ verticalement (cf. figure III-1). Pour un fond plat, cet écho parasite vient donc corrompre la réponse des particules situées à moins d'une distance $\mathrm{d}$ du fond égale à :

$$
\mathrm{d}=\mathrm{P}(1-\cos (\beta))
$$

avec $\beta$ l'angle que forme le faisceau avec la verticale. Pour $\beta=20^{\circ}, 6 \%$ du tirant d'eau sont perdus au fond, et $14 \%$ pour $\beta=30^{\circ}$ [Gordon 1989]. L'angle $\beta$ ne peut cependant pas être trop réduit sans accroître les incertitudes sur la reconstruction géométrique des composantes horizontales de la vitesse.

\section{III.3 HYPOTHÈSE D'HOMOGÉNÉITÉ DU CHAMP DE VITESSE}

Cette hypothèse est peut-être la plus lourde de conséquences sur la qualité de la mesure de vitesse, vu l'écartement rapide des faisceaux et l'ouverture du lobe principal (cf. $\S$ III.2) avec la profondeur, ainsi que la complexité potentielle des écoulements rencontrés en rivière. Pour que la reconstitution géométrique du vecteur vitesse 3D soit valide, il faut en effet que les vitesses radiales mesurées selon chaque faisceau correspondent à un champ de vitesse homogène dans la couche horizontale explorée (Fig. III-2). Dans le cas d'un ADcp à quatre faisceaux, chaque paire de faisceaux diagonaux fournit simultanément une estimation de la composante verticale (w) de la vitesse [RDI 1996]. Si la différence entre ces deux estimations (« error velocity ») est trop grande, on considère que le champ de vitesse est trop perturbé et la mesure est rejetée. Dans le cas d'un aDcp à trois faisceaux, on peut choisir un seuil de variabilité de la vitesse sur le profil vertical comme critère d'hétérogénéité des vitesses. La présence de structures turbulentes d'échelle comparable à l'écartement des faisceaux est une cause probable d'erreurs de mesure inexpliquées. A partir de développements limités du premier ordre, Marsden et al. [2004] proposent une correction de cet effet. 

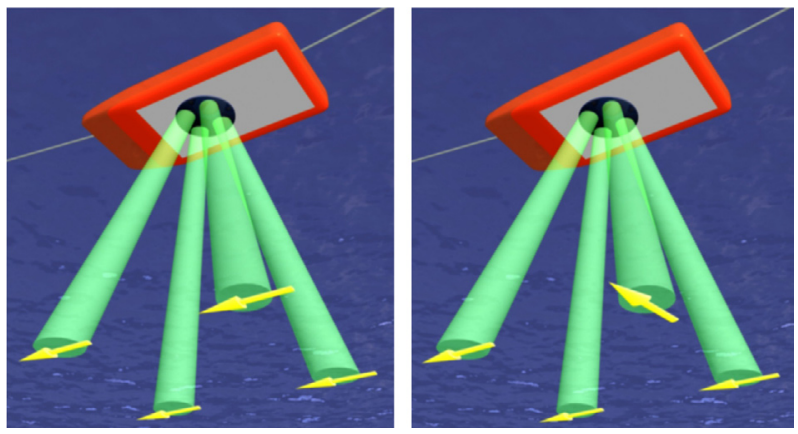

Figure III-2 : champ de vitesse homogène (à gauche) et inhomogène (à droite) dans la couche horizontale interceptée par les faisceaux de l'aDcp

\section{III.4 DÉPLOIEMENT SUR LE TERRAIN}

Le déploiement de l'aDcp sur un cours d'eau peut être rendu difficile par des vitesses élevées, la présence de débris flottants, une surface libre agitée, etc. Son utilisation peut ainsi être limitée lors de fortes crues, ou encore sur des cours d'eau à forte pente. Selon les conditions d'intervention, le support et le mode de déplacement de l'appareil influencent la qualité de la mesure. Des mouvements les plus lents et réguliers possibles, ainsi qu'une minimisation du tangage et du roulis sont préconisés [Yorke et al. 2002].

L'aDcp peut être solidaire d'un bateau, sur le côté ou à l'avant (Fig. III-3), ou bien monté sur un support flottant autonome. Dans ce cas, il peut être maintenu et tracté à l'aide de cordes (depuis un pont ou en travers de la rivière), ou bien motorisé et radiocommandé (l'intérêt principal étant de choisir plus librement la section de mesure, en s'affranchissant des ponts notamment). Les supports non motorisés vont de la planche en mousse au catamaran (kit RiverCat de SonTek, en aluminium) et au trimaran (Riverboat de OceanScience, désormais en polyéthylène haute densité). Du côté des supports motorisés et télécommandés, citons, outre les modèles «maison » conçus par des utilisateurs (par ex. le catamaran de la DIREN Franche-Comté réalisé avec deux pointes de kayak), le monocoque Q-boat commercialisé par OceanScience, le catamaran développé par la société grenobloise Nominal Ingénierie et celui de la société marseillaise AdHoc-Vision, tous deux disponibles en version non motorisée.

La communication entre l'appareil et un ordinateur portable peut se faire soit via un câble, soit à distance (modems radio, Blue-tooth, etc.). La cadence d'acquisition par communication à distance peut parfois être inférieure à la cadence par câble. Le positionnement relatif de l'appareil par suivi acoustique du fond (cf. § IV.1) peut aussi être doublé par GPS embarqué, ce qui est utile en cas de fond mobile. Sinon, l'installation d'un prisme topographique sur l'appareil permet de raccorder aisément les mesures dans un repère absolu à l'aide d'un tachéomètre par exemple. Rarement pratiqué, le déploiement par camion-jaugeur d'aDcp insérés dans un saumon est une piste intéressante pour permettre le jaugeage depuis un pont par fortes vitesses [Lu et al. 2006].

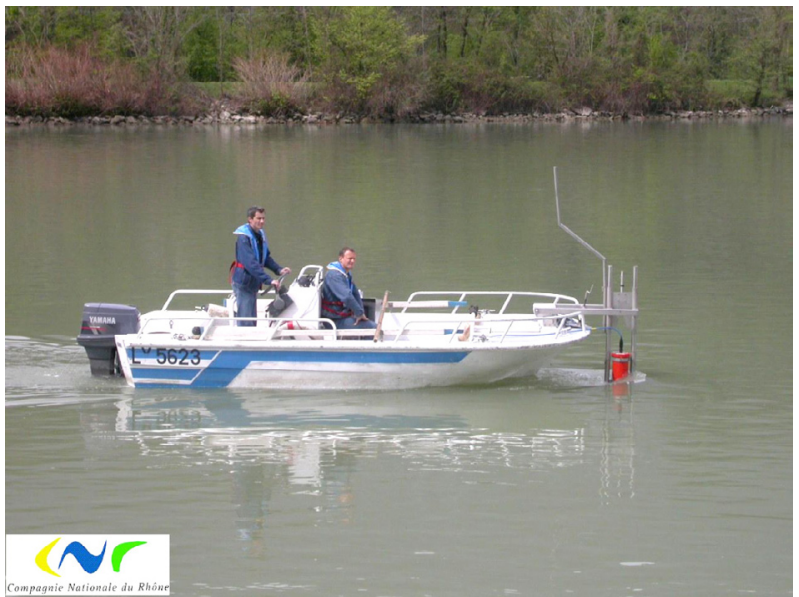

Figure III-3 : déploiement d'un aDcp solidaire d'un bateau (photo CNR)

\section{EXPLOITATION COURANTE DE L'ADCP EN RIVIERE : LE JAUGEAGE}

\section{IV.1 UNE NOUVELLE TECHNIQUE DE JAUGEAGE PLÉBISCITÉE}

Gordon [1989] fut le premier à exposer la possibilité d'utiliser l'aDcp pour le jaugeage d'une rivière (i.e. la mesure du débit total à travers une section), selon le principe du jaugeage par bateau mobile et du suivi de fond (« bottom-tracking »). A l'aide d'impulsions spéciales, l'aDcp calcule à la fois la profondeur et sa propre vitesse par rapport au fond de façon analogue à la mesure de la vitesse d'écoulement (cf. $\S$ II.1). Par composition des vitesses, on obtient sur le profil vertical la vitesse des filets d'eau par rapport au fond. Le tirant d'eau au droit de l'appareil est évalué par la moyenne des profondeurs mesurées par chaque faisceau. On peut donc calculer le débit à travers la surface verticale engendrée par la trajectoire en plan de l'appareil. Ainsi, une traversée de bord à bord, pas nécessairement selon une trajectoire rectiligne ni perpendiculaire aux berges, permet une mesure du débit de la rivière.

En admettant que le champ de vitesse moyen varie peu le temps de la traversée, connaissant $\vec{U}(z, x)$ la vitesse de l'écoulement en fonction de la profondeur $z$ et de la position $x$ le long de la trajectoire, le débit total est par définition :

$$
Q=\iint_{\text {traversée }} \vec{n}(x) \cdot \vec{U}(z, x) d z d x
$$

où $\vec{n}(x)$ est le vecteur unitaire normal à la section en $\mathrm{x}$.

En pratique, l'aDcp utilise la version discrétisée d'une variante de cette équation [Simpson 2001], pour les pas d'espace $\Delta z$ (stipulé par l'opérateur) et $\Delta x$, fonction de la cadence d'acquisition (fonction du paramétrage par l'opérateur) et de la vitesse de déplacement de l'appareil. La mesure du cap de l'aDcp par son compas n'est pas indispensable au calcul du 
débit, grâce au suivi de fond. En revanche, les inclinomètres permettent de limiter les erreurs potentiellement introduites par la non-horizontalité (gîte, assiette) et les mouvements (tangage, roulis) du support.

Cependant, les limitations techniques de l'appareil (cf. partie III) empêchent de connaître la vitesse dans une zone proche de la surface, dans une zone proche du fond, ainsi que près des berges là où la profondeur n'est pas suffisante. Il convient donc d'extrapoler les valeurs de $\vec{U}(z, x)$ dans ces différentes zones. L'usage le plus répandu est d'extrapoler aux bords selon la formule USGS donnée par Simpson [63], de considérer un profil de vitesse en loi-puissance 1/6 au fond [Muste et al. 2004c] et constant près de la surface libre. Le calcul du débit total sur la section (somme du débit mesuré et des différents débits extrapolés) est implémenté dans les logiciels d'acquisition WinRiver (Teledyne RDI, cf. fig. IV-1) et RiverSurveyor (SonTek). Remarquer sur cet exemple que sur $562 \mathrm{~m}^{3} / \mathrm{s}$ estimés, $423 \mathrm{~m}^{3} / \mathrm{s}$ (soit $75 \%$ ) correspondent à des vitesses mesurées, le reste correspondant aux contributions extrapolées, essentiellement en haut des profils verticaux.

Par rapport aux autres techniques de jaugeage (moulinet, courantomètre électromagnétique, dilution chimique, flotteurs [MATE 1998, OMM 1994], mesure des vitesses de surface par vidéo [Creutin et al. 2003], etc.), le jaugeage par aDcp conjugue les avantages de rapidité du jaugeage par bateau mobile (comme par exemple le dispositif soviétique Pless [Klein et al. 1993]) et de précision de la mesure par effet Doppler.

Cette méthode permet ainsi de faire des mesures très rapides, sur des sections de rivières larges et profondes, dans des conditions rendant les techniques traditionnelles coûteuses, dangereuses, voire impossibles : jaugeage du Mississippi pendant la grande crue de 1993 [Admiraal et al. 1996], de l'Amazone et ses affluents [Callède et al. 2000], des différents bras des rivières St-Clair et Detroit dans la région des Grands Lacs [Holtschlag et al. 2002], du Rhône à Beaucaire pendant les crues de novembre 2002 et décembre 2003 (CNR).

Notons enfin qu'outre le jaugeage par aDcp mobile, il est aussi possible de mesurer des profils verticaux de vitesse en des points fixes de la section, comme lors d'un jaugeage classique au courantomètre (logiciel Stationary Measurement de SonTek et logiciel Section-by-Section de Teledyne RDI). Cette méthode peut s'avérer utile dans certaines conditions, notamment en cas de fonds mobiles (cf. § IV.3) ou sur des rivières gelées où l'on creuse des trous dans la glace, au niveau des verticales de mesure [Mudge et al. 2005].

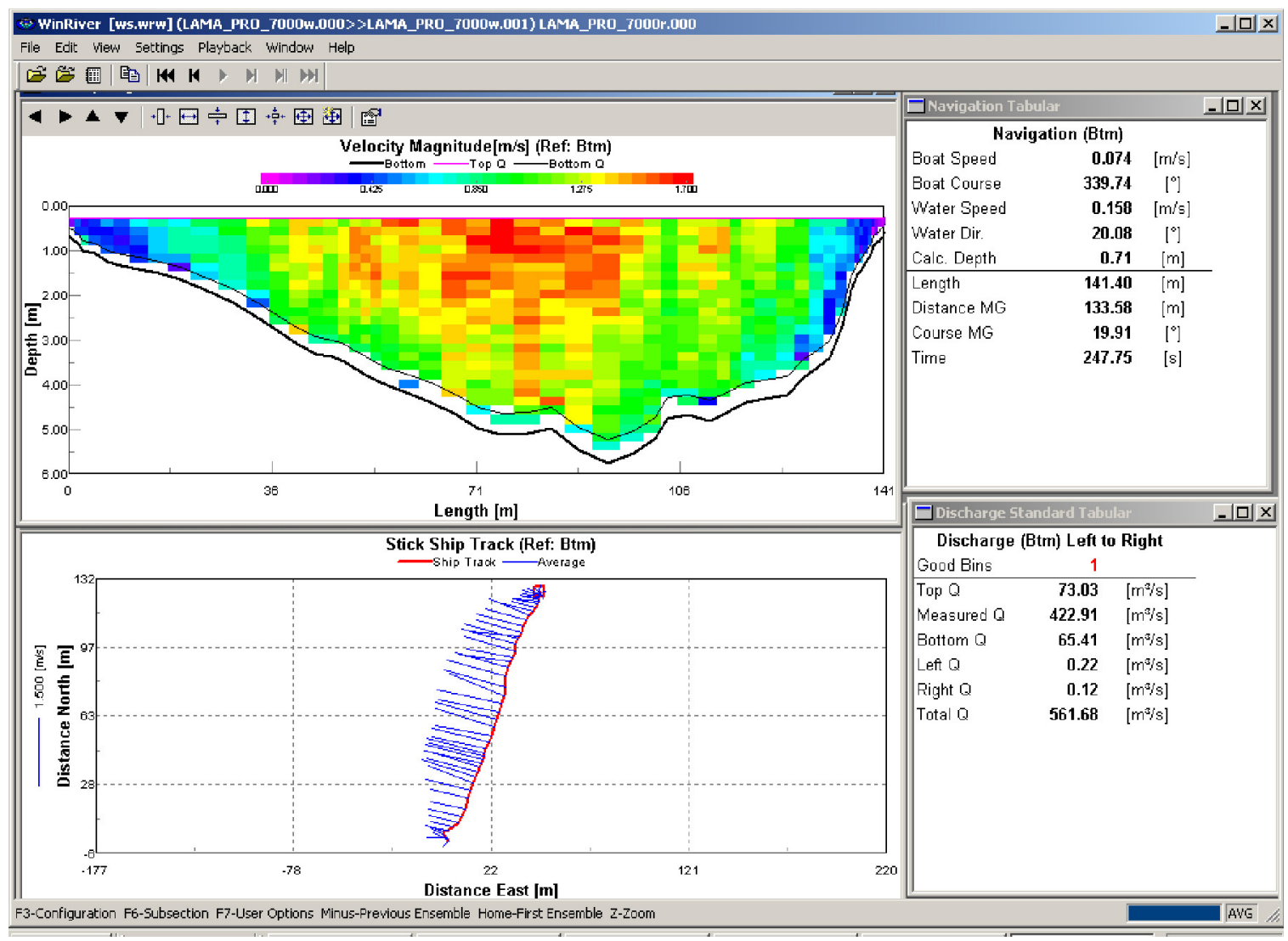

Figure IV-1 : jaugeage de la Garonne par ADCP RDI Rio Grande $1200 \mathrm{kHz}$ (Cemagref, Lyon) - profils d'intensité de vitesse (haut) - trajectoire et vitesses moyennes sur la verticale (bas) 


\section{IV.2 EVALUATION DE LA QUALITÉ DE LA MESURE}

Avant la toute récente publication de la norme ISO/TS $24154: 2005$, les mesures de débit par aDcp mobile ne faisaient l'objet d'aucunes préconisations NF ou ISO. Dans la littérature scientifique, généralement la répétabilité des jaugeages par aDcp varie entre $1 \%$ (Filizola et al. [2004], Amazone) et $5 \%$ (Admiraal et al. [1996], Mississippi en crue) et l'erreur totale sur le débit est évaluée à $5 \%$ ou moins (Gordon [1989] sur l'Elbe, Filizola et al. [1999] sur la Solimões).

La confrontation du débit obtenu par $\mathrm{aDcp}$ à des mesures classiques est souvent pratiquée et commence à être documentée. En particulier, l'accord avec les jaugeages au courantomètre est typiquement inférieur à $5 \%$, quel que soit le débit, la marque et le type de l'appareil [Mueller 2002, Terek 2004, Pierrefeu 2004]. Plus rarement, l'écart peut s'élever à $10 \%$ dans le cas d'instabilités hydrauliques [Pierrefeu 2004].

Ces vérifications de l'aDcp in situ sont rassurantes, mais elles comportent deux limites. D'abord, aucune mesure du débit, même par exploration des vitesses, ne peut être prise comme étalon: la précision communément admise pour le jaugeage au courantomètre est de l'ordre de $7 \%$, sur la base d'essais en laboratoire. Ensuite, les explorations des vitesses par courantomètre ou par aDcp n'induisent pas les mêmes moyennes spatiales et temporelles, les deux débits élaborés ne sont donc pas de natures strictement équivalentes.

Les essais en conditions contrôlées permettent de vérifier et d'étalonner les mesures acoustiques brutes (vitesse de l'eau, vitesse du fond, bathymétrie). Ils consistent généralement à remorquer l'appareil à vitesse constante au dessus d'un bassin d'eau immobile, la principale difficulté technique étant de disposer de suffisamment de particules en suspension pour permettre la rétrodiffusion des ultrasons. Lors de tels tests réalisés par l'USGS sur des ADCP Teledyne RDI à $600 \mathrm{kHz}$ et $1200 \mathrm{kHz}$, la différence moyenne entre la vitesse de traction et la vitesse mesurée par l'aDcp (erreur systématique ou biais) était de $-0,21 \mathrm{~cm} / \mathrm{s}$ et $-0,23 \mathrm{~cm} / \mathrm{s}$ pour le suivi du fond et le suivi de l'eau respectivement, pour une gamme de vitesses de 0 à $2,5 \mathrm{~m} / \mathrm{s}$ [Oberg 2002]. La précision des mesures brutes non acoustiques (temps, température, inclinaison...) est également à prendre en compte.

Toutefois, ces tests ne reflètent pas les conditions d'utilisation en milieu naturel, pour lesquelles des sources d'erreur supplémentaires interviennent : fond mobile, perte de l'écho de fond, mouvements du support, écoulements complexes et inhomogénéité des vitesses, etc. Il est difficile de faire des tests en canal de laboratoire avec des tirants d'eau suffisants. L'USGS a toutefois pu comparer des mesures de vitesses proches de $1 \mathrm{~m} / \mathrm{s}$ par aDcp et par aDv dans un canal d'irrigation [Gartner et al. 2002].

Par ailleurs, une fois estimées les incertitudes sur les mesures brutes, il reste à les propager dans la chaîne de calcul complexe du débit total [Muste et al. 2004a]. Dans ce processus, il faut tenir compte des moyennes spatiales et temporelles à l'œuvre, réduisant notamment la dispersion des mesures (erreur aléatoire). Ce «bruit Doppler» peut aller de quelques $\mathrm{mm} / \mathrm{s}$ à $0,5 \mathrm{~m} / \mathrm{s}$ en fonction du type d'appareil, de la fréquence du signal, de la taille de cellule, etc. [RDI 1996]. L'écart-type d'erreur d'estimation de la moyenne de $\mathrm{N}$ mesures est inversement proportionnel à racine de $\mathrm{N}$ si l'on fait l'hypothèse que les erreurs des $\mathrm{N}$ mesures sont indépendantes. Moyenner les mesures ne résorbera toutefois pas une éventuelle erreur systématique.

En attendant la formalisation du calcul des incertitudes liées à la mesure de débit par aDcp, les utilisateurs recherchent un cadre de bonnes pratiques leur permettant d'assurer la qualité d'une mesure, selon les conditions rencontrées. Les recommandations de l'USGS [Lipscomb 1995, Oberg et al. 2005] s'appuient en effet sur la répétabilité de quatre mesures successives pour éliminer a posteriori tout résultat s'écartant de plus de $5 \%$ de la moyenne, à condition de trouver une cause plausible de perturbation de la mesure. Sinon, il faut réaliser quatre nouvelles traversées et moyenner les huit.

\section{IV.3 FOND MOBILE}

Quand le lit du cours d'eau est mis en mouvement sous l'effet de la contrainte au fond (situations de charriage mais aussi de lit fluidisé), les vitesses d'écoulement et la trajectoire estimées par l'aDcp présentent un biais. Plusieurs expérimentateurs ont signalé et étudié cette erreur systématique entre la trajectoire calculée par l'aDcp et la trajectoire réelle de l'appareil, qu'elle soit obtenue par levé au tachéomètre, par GPS, ou estimée par retour à une position fixe après deux traversées. Ce biais peut également provenir des sédiments transportés en suspension à proximité du fond (cf. $\S$ V.4).

Sur des sections de grands cours d'eau amazoniens, Callède et al. [2000] observent ainsi une dérive de la trajectoire apparente de leur aDcp vers l'amont, de même que la CNR sur le Rhône [Pierrefeu 2004]. Dans ce cas, le plus courant, l'appareil sous-estime systématiquement les vitesses d'écoulement et donc le débit total sur la section. Mais sous l'influence de la marée montante, le lit de la Loire près de Nantes peut par exemple être mis en mouvement vers l'amont (DIREN Centre, comm. pers.). L'USGS préconise des tests pour détecter un éventuel déplacement du fond, mais ne détaille pas de méthode de correction du débit [Oberg et al. 2005].

Deux méthodes pour corriger cet effet sont envisageables : - Erreur de position de retour : en estimant sur chaque allerretour l'erreur de position calculée pour un même point de départ et d'arrivée, on peut estimer la vitesse moyenne $\mathrm{du}$ fond sur l'ensemble de la section [Callède et al. 2000, Pierrefeu 2004].

- Erreur de position instantanée : si l'aDcp est fixe ou si on connaît la trajectoire réelle de l'aDcp (par GPS par exemple), on peut calculer l'erreur de trajectoire en chaque point de la section, et donc évaluer la vitesse du fond localement sur chaque verticale. Une évaluation du transport de la charge de fond peut être tentée sur ce principe (cf. § V.4).

Notons que la proximité d'objets magnétiques peut aussi donner lieu à des trajectoires apparentes aberrantes en faussant la mesure du compas interne de l'aDcp [Admiraal et al. 1996]. 


\section{$V \square$ VERS D'AUTRES TYPES D'EXPLOITATION DE L'ADCP}

Au-delà de l'application au jaugeage des cours d'eau, de nombreux efforts de recherche sont actuellement en cours pour évaluer la possibilité d'utiliser l'aDcp pour des mesures de bathymétrie, de champ de vitesse et de transport de sédiments [Dinehart et al. 2005a, 2005b, Kostaschuk et al. 2005].

\section{V.1 BATHYMÉTRIE}

Le suivi du fond par l'aDcp fournit en général des profils bathymétriques qui présentent une bonne répétabilité sur plusieurs traversées [Admiraal et al. 1996], et sont de plus en plus souvent exploités comme une donnée indépendante. Ainsi, Strasser et al. [2002] ont exploité les données bathymétriques et les profils de vitesses établis par aDcp (300 et $600 \mathrm{kHz}$ ) pour étudier la géométrie des dunes du moyen Amazone en fonction des caractéristiques de l'écoulement.

Ces traversées peuvent être raccordées à un repère absolu par des levés au tachéomètre, en début et en fin d'enregistrement par exemple (l'installation d'un prisme topographique sur l'appareil ou son support facilite l'opération). Le recours à un GPS embarqué permet de même de s'affranchir de l'éventuelle dérive due à un fond mobile (cf. § IV.3).

Plutôt que de se contenter de la moyenne arithmétique des profondeurs mesurées par chacun des faisceaux [RDI 1996], il est possible de repositionner et d'exploiter directement la mesure de profondeur effectuée par chaque faisceau, en compensant éventuellement tangage et roulis [Dinehart et al. 2005a, 2005b].

\section{V.2 MESURE DU CHAMP DE VITESSE MOYEN 3D}

L'aDcp permet une exploration très rapide des écoulements en rivière, sur des domaines vastes. Les distributions de vitesse moyenne ainsi obtenues peuvent servir au calage et à l'évaluation de modèles hydrodynamiques [Kolb 1995, Rubbert et al. 2003, Védie 2005]. Avec un aDcp flottant ancré dans un écoulement bidimensionnel et stationnaire (canal de navigation), Gonzalez et al. [1996] parviennent à un bon accord des profils de vitesse expérimentaux avec les lois théoriques logarithmique ou puissance 1/6.

Au-delà du profil vertical des vitesses ou de leur distribution en plan, l'identification des structures persistantes de l'écoulement (recirculations, couches de mélange, courants secondaires hélicoïdaux, etc.) sont quantifiables par mesure aDcp, à condition que leurs échelles caractéristiques soient supérieures au volume d'échantillonnage [Muste et al. 2004b]. L'aDcp permet ainsi d'observer les structures de l'écoulement (cf. fig. V-1), qui conditionnent l'évolution morphodynamique des rivières, que ce soit au niveau des méandres [Dinehart et al. 2005a], des confluences [Dinehart et al. 2005b], des bifurcations [Richardson et al. 1996], des champs de dunes [Parsons et al.2005], des annexes hydrauliques [Védie 2005], etc. L'aDcp peut aussi aider à la caractérisation de l'hétérogénéité hydrodynamique - et donc de la richesse biologique - des habitats aquatiques [Shields 2003, 2005]
Certains utilisateurs développent des outils informatiques pour interpoler, superposer et comparer les profils de vitesse sur plusieurs traversées [Adler et al. 2001, Dinehart et al. 2005a, 2005b, Kim et al. 2005, Muste et al. 2004c, Le Coz et al. 2006].

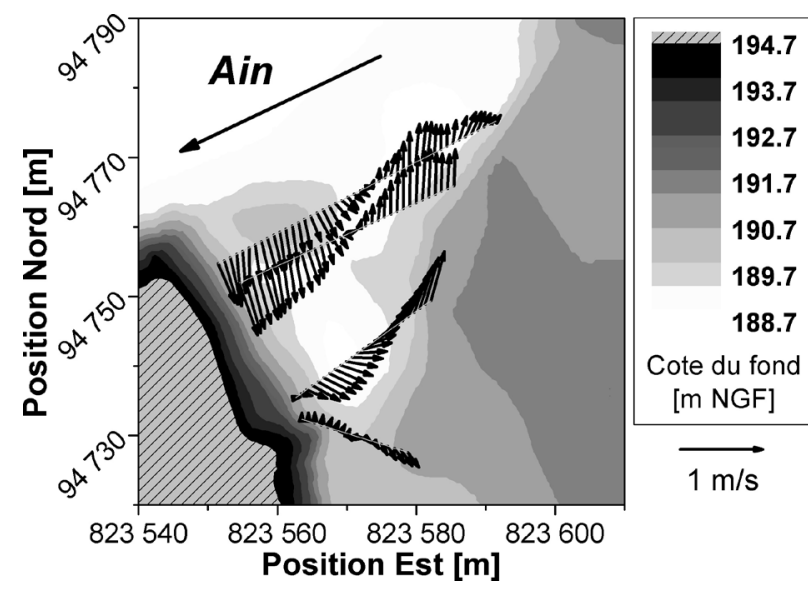

Figure V-1 : distribution des vitesses intégrées sur la verticale à l'aval d'un bras mort de l'Ain

\section{V.3 MESURE DU CHAMP INSTANTANÉ ET DES CARACTÉRISTIQUES TURBULENTES}

Dans de nombreux travaux récents en milieu marin et estuarien, l'aDcp est utilisé pour mesurer les paramètres de turbulence des écoulements (voir Lu et al. [1999] par exemple). Une première approche consiste à supposer que les seconds moments des fluctuations turbulentes sont homogènes sur les volumes d'échantillonnage des quatre faisceaux et à en déduire les composantes du tenseur de Reynolds [Schemper et al. 2002, Stacey et al. 1997]. L'accès à l'énergie cinétique turbulente $k$ nécessite alors d'évaluer l'anisotropie de la turbulence, soit à l'aide de constantes empiriques données par exemple par Nezu et al. [1993], soit en mesurant directement les fluctuations de la vitesse verticale w'à l'aide d'un cinquième faisceau vertical [Lu et al. 1999]. La contribution du «bruit Doppler» lié à l'appareil doit dans tous les cas être évaluée [Lu et al. 1999] et éventuellement compensée [Hurther et al. 2001]. En outre, dans le cas d'un $\mathrm{aDcp}$ lié à un support flottant, et non fixé rigidement au fond ou sur un dispositif de laboratoire, les mouvements de rotation (cap, tangage, roulis) sont une source d'erreurs potentiellement assez grandes [Lu et al. 1999] pour compromettre cette première méthode en l'absence de correction.

Muste et al. [2004b] ont considéré que les tourbillons les plus énergétiques ont des tailles supérieures à l'écartement des faisceaux et ont étudié la turbulence grandes échelles. En faisant l'hypothèse d'homogénéité des vitesses par couches horizontales dans le champ des faisceaux, l'intensité turbulente peut être calculée à partir des fluctuations de la vitesse mesurée et reconstituée par l'aDcp [Barua et al. 1998, Muste et al. 2004b]. En régime permanent, vitesse 
moyenne et intensité turbulente se stabilisent pour des séries de mesure suffisamment longues, typiquement $15 \mathrm{~min}$ pour une fréquence de mesure de l'ordre du Hertz [Barua et al. 1998, Gonzalez-Castro et al. 2002, Muste et al. 2004b].

Des travaux complémentaires sont nécessaires pour préciser dans quelle mesure la turbulence grandes échelles est observable efficacement par aDcp en rivière. En tout état de cause, l'analyse fine de la turbulence permise par les mesures ponctuelles à haute cadence des $\mathrm{aDv}$ reste hors de portée des aDcp du commerce [Nystrom et al. 2002]. Fonctionnant en bistatique et en mode cohérent, le profileur acoustique expérimental ADVP [Lemmin et al. 1997] est capable de fournir des mesures de turbulence s'il est fixé sur une structure immobile, par exemple sur un cours d'eau très peu profond équipé d'une passerelle métallique [Franca 2005].

\section{V.4 MESURE DU TRANSPORT DE LA CHARGE DE FOND}

Le suivi de fond (bottom-tracking) par un aDcp fixe ou mobile et positionné par GPS centimétrique permet d'obtenir la vitesse réelle de déplacement du fond (cf. § IV.3), dont dépend le transport par charriage. De tels essais ont été menés sur des rivières à galets [Rennie et al. 2002] et à sable [Gaeuman et al. 2006, Villard et al. 2005]. Dans ce dernier cas, les mesures instantanées apparaissent moins dispersées, sans doute parce que le transport de fond est plus homogène et continu [Villard et al. 2005].

La principale difficulté vient du fait que la vitesse de fond mesurée par profileur Doppler est une moyenne sur les contributions respectives de la couche active et des couches immobiles plus profondes, voire de la forte charge en suspension près du fond ("water bias", cf. [RDI 1996]). En outre, plus la fréquence est basse plus la profondeur de pénétration des ultrasons dans les couches de sédiments croît. Des mesures conjointes montrent qu'un aDcp à 1500 $\mathrm{kHz}$ peut détecter un fond mobile quand un $\mathrm{aDcp}$ à $300 \mathrm{kHz}$ mesure une vitesse de fond nulle, ses impulsions pénétrant jusqu'aux couches immobiles [Rennie et al. 2004].

Pour évaluer le transport de fond à partir de la vitesse de fond, il faut estimer la porosité et l'épaisseur de la couche active à partir d'hypothèses souvent simplifiées. Les taux de transport estimés par aDcp sont alors en bon accord avec ceux mesurés par des échantillonneurs traditionnels [Rennie et al. 2002, Villard et al. 2005].

\section{V.5 MESURE DES FLUX DE MATIÈRES EN SUSPENSION}

Parmi les différentes méthodes de mesure du transport de matières en suspension (MES), les technologies acoustiques semblent les plus prometteuses pour l'avenir [Wren et al. 2000]: non intrusives, elles ont l'avantage de fournir simultanément et au même endroit des informations sur la topographie du fond, le champ de vitesse, les concentrations en MES voire leur granulométrie (cf. Thorne et al. [2002] par exemple).

Les contrastes temporels et spatiaux de taux de MES au sein des masses d'eau explorées peuvent être caractérisés à partir des profils d'intensité du signal acoustique rétrodiffusé.
Cette interprétation de l'écho peut être qualitative [Dinehart et al. 2005a, Richardson et al. 1996], ou bien fondée sur des corrélations empiriques établies à partir de prélèvements : Holdaway et al. [1999] (en milieu estuarien), Filizola et al. [2004] (sur l'Amazone), Kostaschuk et al. [2005] (en milieu lacustre), Lorke et al. [2004] (zooplancton lacustre), Shen et al. [1999] (en laboratoire). En milieu côtier, la comparaison avec des concentrations mesurées par aDcp et celles issues de transmissomètres (méthode optique indirecte) a donné de bons résultats [Gartner 2004, Hill et al. 2003, Hoitink 2004, Hoitink et al. 2005, Holdaway et al. 1999].

On peut aussi expliciter les relations théoriques qui régissent la rétrodiffusion du signal acoustique par les particules en suspension dans l'eau [DRL 2003, Holdaway et al. 1999, Land et al. 2001, Thorne et al. 2002]. L'interprétation de l'intensité de l'écho en termes de concentration dépend fondamentalement des variations de granulométrie au sein de l'écoulement [Gartner 2004, Kostaschuk et al. 2005, SonTek 1997]. L'aDcp, appareil monofréquence, ne permet de mesurer que la concentration des sédiments, sans informations sur leur distribution granulométrique [Reichel et al. 1994] à la différence des systèmes à rétrodiffusion acoustique multifréquence utilisés en océanographie (cf. Taylor et al. [1998] par exemple).

L'aDcp apparaît potentiellement comme une solution alternative d'exploration et de jaugeage des MES, sur les grands cours d'eau surtout, mais des prélèvements réalisés en parallèle restent nécessaires pour obtenir des concentrations absolues. L'ensemble des facteurs d'incertitude environnementaux à prendre en compte pour l'application aux cours d'eau reste encore peu documenté [Rotaru et al. 2006].

\section{VI $\square$ CONCLUSION}

LaDcp représente une forte innovation technologique pour l'hydrométrie, en remplacement ou en complément des techniques classiques de jaugeage par exploration du champ de vitesse (moulinets, courantomètres électromagnétiques, etc.). Bien entendu, ce n'est pas la panacée universelle du jaugeur, notamment face aux torrents, aux berges inaccessibles et aux crues violentes. Le profileur acoustique Doppler offre en outre la possibilité d'accéder rapidement à un semis bathymétrique et au champ de vitesse tridimensionnel. Enfin il offre d'intéressantes potentialités pour la mesure du transport de sédiments, aussi bien par charriage qu'en suspension, moyennant cependant des efforts de recherche et développement.

L'estimation des incertitudes de mesure de débit n'est pas encore totalement maîtrisée, mais la plupart des comparaisons empiriques avec des méthodes classiques donnent satisfaction. En attendant, les jaugeurs ont commencé à établir des modes opératoires et un savoir faire pour garantir la qualité de leurs mesures et cerner les cas où l'aDcp est inadapté. C'est à la fois en évaluant le comportement de l'appareil en conditions variées (nature du fond et des particules rétrodiffusantes, caractéristiques de l'écoulement et du transport solide, modes d'acquisition, etc.), en analysant le détail de son fonctionnement et en explorant toutes ses potentialités que l'on pourra encore mieux l'utiliser et le maîtriser. Retour d'expérience opérationnel et essais expérimentaux apparaissent ici complémentaires. 


\section{VII — REMERCIEMENTS}

L'acquisition d'un ADCP Teledyne RDI Rio Grande WorkHorse $1200 \mathrm{kHz}$ par l'UR HHLY (Cemagref) a été soutenue par le Cemagref, par le programme «Modifications anthropiques des flux sédimentaires des cours d'eau, réponses des écosystèmes aquatiques et actions de restauration » de la Région Rhône-Alpes et par le programme national ACI «ECCO » $\mathrm{PNRH}$, « étude du fonctionnement hydro-sédimentaire des annexes fluviales ». Merci à C. Haritchabalet, E. Hérouin, D. Hurther, L. Michel, A. Olivier et G. Pierrefeu pour leur relecture attentive des premières versions de cet article. Un grand merci enfin à A.-L. Achard, M.-P. Baligand et A. Bazergan, documentalistes au Cemagref, pour leur contribution extrêmement efficace à la collecte des publications citées.

\section{VIII — REFERENCES}

[1] Norme ISO/TS 24154 :2005 2005. Hydrometry - Measuring river velocity and discharge with acoustic Doppler profilers, $10 \mathrm{p}$.

[2] Adler M. \& Nicodemus U.2001. A new computer model for the evaluation of data from acoustic Doppler current profilers (ADCP). Physics and Chemistry of the Earth, Part C: Solar, Terrestrial and Planetary Science, 26 (10-12), 711-715.

[3] Admiraal D. \& Demissie M. 1996. Velocity and discharge measurements at selected locations on the Mississippi river during the Great Flood of 1993 using an Acoustic Doppler Current Profiler. Water International, 21, 144-151.

[4] Barua D.K., \& Rahman K.H.1998. Some aspects of turbulent flow structure in large alluvial rivers. Journal of Hydraulic Research, 36 (2), 235-252.

[5] Birgand F., Benoist J.-C., Novince E., Gilliet N., SaintCast P., \& Le SaOs E.2005. Mesure des débits à l'aide de débitmètres ultrasoniques Doppler - Cas des petits cours d'eau ruraux. Ingénieries - EAT, 41, 23-38.

[6] Callède J., Kosuth P., Guyot J.L., \& Santos Guimaraes V.2000. Discharge determination by ADCP : a moving bottom error correction method and its application on the river Amazon at Obidos. Hydrological Sciences Journal, 45 (6), 911924.

[7] Cheng R. T. \& Gartner J. W.2003. Complete velocity distribution in river cross-sections measured by acoustic instruments. IEEE/OESS 7th Working Conference on Current Measurement Technology, San Diego, California, 21-26.

[8] Creutin J.D., Muste M., Bradley A.A., Kim S.C. \& Kruger A.2003. River gauging using PIV techniques : a proof of concept experiment on the Iowa river. Journal of Hydrology, 277 (3-4), 182-194.

[9] Dinehart R.L., \& Burau J.R. 2005a. Averaged indicators of secondary flow in repeated acoustic Doppler current profiler crossings of bends. Water Resources Research, 41 (9), 1-18.

[10] Dinehart R.L. \& Burau J.R. 2005b. Repeated surveys by acoustic Doppler current profiler for flow and sediment dynamics in a tidal river. Journal of Hydrology, 314 (1-4), 1-21.

[11] Doppler C.1842. Über das farbige Licht der Doppelsterne und einiger anderer Gestirne des Himmels (Sur la lumière colorée des étoiles doubles et d'autres étoiles du ciel). Abhandlungen der Königlich Böhmischen Gesellschaft der Wissenschaften, 2 , 465-482.

[12] DRL 2003. DRL-Sediview software user manual V3, DRL Software Ltd.

[13] Filizola N., Guimaraes V. \& Guyot J.L. 1999. Medição de vazão em grandes rios com o uso do perfilador Doppleracustico de corrente. Manaus'99 international symposium Hydrological and Geochemical processes in large scale river basins. Manaus, Brasil.

[14] Filizola N. \& Guyot J.L. 2004. The use of Doppler technology for suspended sediment discharge determination in the river Amazon. Hydrological Sciences Journal, 49 (1), 143-153.

[15] Franca M.J.2005. Flow dynamics over a gravel riverbed. XXXI IAHR congress. Seoul, Korea, 6542-6551.

[16] Gaeuman D. \& Jacobson R.B.2006. Acoustic bed velocity and bed load dynamics in a large sand bed river. Journal of Geophysical Research-Earth Surface, 111(F2).

[17] Gartner J.W. \& Ganju N.K.2002. A preliminary evaluation of near-transducer velocities collected with low-blank ADCP, Hydraulic Measurements and Experimental Methods 2002. Estes Park, Colorado, USA

[18] GARTNER J.W.2004. Estimating suspended solids concentrations from backscatter intensity measured by acoustic Doppler current profiler in San Francisco Bay, California. Marine Geology, 211 (3-4), 169-187.

[19] Gonzalez J.A., Melching C.S. \& Oberg K.A.1996. Analysis of open-channel velocity measurements collected with an Acoustic Doppler Current Profiler. 1st International Conference on New/Emerging Concepts for Rivers, I. W. R. Association. Chicago, USA.

[20] Gonzalez-Castro J.A., Oberg K.A., Duncker J.J.2002. Effect of temporal resolution on the accuracy of ADCP measurements, Hydraulic Measurements and Experimental Methods 2002. Estes Park, Colorado, USA.

[21] GoRdOn R.L. 1989. Acoustic measurement of river discharge. Journal of Hydraulic Engineering, 115 (7), 925-936.

[22] Hill D.C., Jones S.E. \& Prandle D.2003. Derivation of sediment resuspension rates from acoustic backscatter time-series in tidal waters. Continental Shelf Research, 23, 19-40.

[23] HoitinK A.J.F. 2004. Tidally-induced clouds of suspended sediment connected to shallow-water coral reefs. Marine Geology, 208 (1), 13-31.

[24] Hoitink A.J.F. \& Hoekstra P. 2005. Observations of suspended sediment from ADCP and OBS measurements in a muddominated environment. Coastal Engineering, 52, 103-118.

[25] Holdaway G.P., Thorne P.D., Flatt D., Jones S.E. \& Prandle D.1999. Comparison between ADCP and transmissometer measurements of suspended sediment concentration. Continental Shelf Research, 19, 421-441.

[26] Holtschlag D.J. \& KoschiK J.A.2002. Flow distribution in selected branches of St. Clair and Detroit rivers. Journal of Great Lakes Research, 28 (3), 379-395.

[27] HURTHER D. \& Lemmin U.2001. A correction method for turbulence measurements with a 3D Acoustic Doppler Velocity Profiler. Journal of Atmospheric and Oceanic Technology, 18, 446-458. 
[28] Kim D., Muste M. \& Weber L. 2005. Development of new ADCP post-processing and visualization capabilities. XXXIth IAHR Congress, Séoul, Corée, 338-347.

[29] KLein G.S., Yufit G.A. \& ShKURKo V.K.1993. A new moving boat method for the measurement of discharge in large rivers. Hydrological Sciences Journal, 38 (2), 79-88.

[30] KoLB M. 1995. Experiences with vessel-borne ADCPs in shallow waters. IEEE 5th working conference on current measurement. St. Petersburg, Florida, USA, 79-82.

[31] Kostaschuk R., Best J., Villard P., Peakall J. \& Franklin M. 2005. Measuring flow velocity and sediment transport with an acoustic Doppler current profiler. Geomorphology, 68, 2537.

[32] LAND J.M. \& JoNES P.D.2001. Acoustic measurement of sediment fluxes in rivers and near-shore waters. Seventh Federal Interagency Sedimentation Conference. Reno, Nevada.

[33] Le Coz J., Hauet A., Védie F., Dramais G., Chastan B. \& PAQUIER A.2006. Recirculating flow pattern assessment from aDcp, LS-PIV and 2Dh modelling. RiverFlow2006. Lisbon, Portugal, 2, 1843-1851.

[34] Lemmin U. \& Rolland T.1997. Acoustic velocity profiler for laboratory and field studies. Journal of Hydraulic Engineering, 123 (12), 1089-1098.

[35] Lipscomb S.W.1995. Quality assurance plan for discharge measurements using broadband acoustic Doppler current profilers, USGS open-file report 95-701, $5 \mathrm{p}$.

[36] Lorke A., McGinNis D.F., SpaAk P., \& Wuest A.2004. Acoustic observations of zooplankton in lakes using a Doppler current profiler. Freshwater Biology, 49, 1280-1292.

[37] LU Y. \& LUECK R.G.1999. Using a broadband ADCP in a tidal channel. Part II : Turbulence. Journal of Atmospheric and Oceanic Technology, 16 (11), 1568-1579.

[38] Lu J.-Y. \& Su C.-C. et WANG C.-Y.2006. Application of a portable measuring system with acoustic Doppler current profiler to discharge observations in steep rivers. Flow Measurement and Instrumentation17(3), 179-192.

[39] Marsden R.F. \& Ingram R.G.2004. Correcting for beam spread in acoustic Doppler current profiler measurements. Journal of Atmospheric and Ocean Technology, 21 (9), 14911498.

[40] MATE1998. Charte qualité de l'hydrométrie - Code de bonnes pratiques, Ministère de l'Aménagement du Territoire et de l'Environnement, $50 \mathrm{p}$.

[41] Mueller D.S.2002. Field evaluation of boat-mounted acoustic Doppler instruments used to measure streamflow, Hydraulic Measurements and Experimental Methods 2002. Estes Park, USA.

[42] Muste M., Yu K., Gonzalez-Castro J.A., Ansar M. \& Startzman R. 2004a. Methodology for estimating ADCP measurement uncertainty in open-channel flows. World water congress 2004 : critical transitions in water and environmental resources management. Salt Lake City, Utah, USA.

[43] Muste M., Yu K., Pratt T. \& Abraham D.2004b. Practical aspects of ADCP data use for quantification of mean river flow characteristics ; Part II : fixed-vessel measurements. Flow Measurement and Instrumentation, 15, 17-28.

[44] Muste M., Yu K. \& Spasojevic M. 2004c. Practical aspects of ADCP data use for quantification of mean river flow characteristics ; Part I : moving-vessel measurements. Flow Measurement and Instrumentation, 15, 1-16.

[45] Nezu I. \& NaKagawa H.1993. Turbulence in open-channel flows. Rotterdam, IAHR Monograph.

[46] Nystrom E.A., Oberg K.A. \& Rehmann C.R. 2002. Measurement of turbulence with acoustic doppler current profilers - sources of error and laboratory results. Hydraulic Measurements and Experimental Methods 2002. Estes Park, Colorado, USA.

[47] Oberg K.A.2002. In search of easy-to-use methods for calibrating ADCP's for velocity and discharge measurements, Hydraulic Measurements and Experimental Methods 2002. Estes Park, Colorado, USA.

[48] Oberg K.A., Morlock S.E. \& Caldwell W.S.2005. Qualityassurance plan for discharge measurements using acoustic Doppler current profilers, USGS open-file report 2005-5183, $35 \mathrm{p}$.

[49] OMM1994. Guide des Pratiques Hydrologiques, Organisation Météorologique Mondiale.

[50] Parsons D., Best J., Orfeo O., Hardy R.J., Kostaschuk R. \& LANE S.N.2005. Morphology and flow fields of three-dimensional dunes, Rio Parana, Argentina : Results from simultaneous multibeam echo sounding and acoustic Doppler current profiling. Journal of Geophysical Research-Earth Surface, 110(F4).

[51] Pierrefeu G.2004. Effects of a moving bottom on a discharge measurement. Discharge measurements using a DGPS. RD Instruments ADCPs in Action - Europe. Nice, France.

[52] RDI1996. Acoustic Doppler Current Profiler - Principles of operation - A practical primer. San Diego, California, RD Instruments.

[53] Reichel G. \& Nachtnebel H.P.1994. Suspended sediment monitoring in a fluvial environment: advantages and limitations applying an acoustic Doppler current profiler. Water Research, 28 (4), 751-761.

[54] Rennie C.D., Millar R.G. \& Church M.A.2002. Measurement of bed load velocity using an ADCP. Journal of Hydraulic Engineering, 128 (5), 473-483.

[55] RenNie C.D. \& Millar R.G.2004. Measurement of the spatial distribution of fluvial bedload transport velocity in both sand and gravel. Earth Surface Processes and Landforms, 29, 11731193.

[56] Richardson W.R., Thorne C.R. \& Mahmood S.1996. Secondary flow and channel changes around a bar in the Brahmaputra river, Bangladesh. Coherent flow structures in open channels, Ashworth ed., 519-543.

[57] Rotaru E., Le Coz J., Drobot R., Adler M.-J., Dramais G.2006. ADcp measurements of suspended sediment fluxes in Banat rivers, Romania. Balwois2006, Ohrid, Macedonia.

[58] Rubbert S., Van Linn A., Rettemeier K. \& Kongeter J.2003. Comparison of ADCP measurements and 3D large-eddybased simulations of flow in small, shallow Quitzdorf reservoir. Water Engineering and Research in a Learning Society : Modern Developments and Traditional Concepts; XXX IAHR Congress. Thessaloniki, Greece.

[59] SChemper J.S. \& Admiraal D.M. 2002. An examination of the application of ADCP measurements in a wide channel of uniform depth for turbulence calculations. Hydraulic Measurements and Experimental Methods. Estes Park, Colorado, USA. 
[60] Shen C. \& Lemmin U.1999. Application of an acoustic particle flux profiler in particle-laden open-channel flow. Journal of Hydraulic Research, 37 (3), 407-419.

[61] SHIELDS F.D.J.2003. Use of acoustic Doppler current profilers to describe velocity distributions at the reach scale. Journal of the American Water Resources Association, 39 (6), 1397-1408.

[62] SHIELDS F.D.J.2005. River habitat quality from river velocities measured using Acoustic Doppler Current Profiler. Environmental Management, 36 (4), 565-575.

[63] Simpson M.R. 2001. Discharge measurements using a broadband acoustic Doppler current profiler. Sacramento, California, USGS open-file report 01-1, $123 \mathrm{p}$.

[64] SonTek1997. SonTek Doppler current meters - Using signal strength data to monitor suspended sediment concentration.

[65] SonTek2001. Principes de fonctionnement du PC-ADP, SonTek/YSI - AnHydre.

[66] Stacey M.T. \& Monismith S.G.1997. Measuring estuarine turbulence with an ADCP. Congress of the IAHR, B1, 155-160.

[67] Strasser M.A., Vinzon S.B. \& Kosuth P. 2002. Bottom structures geometry of the Amazon river. RiverFlow 2002. Louvainla-Neuve, Belgium, 2, 1185-1193.

[68] Taylor J.A., Vincent C.E., Thorne P.D., Hardcastle P.J., Humphrey V.F., Zhang J.D., Schaafsma A., Dohmen-Janssen C.M. \& Perennes M. 1998. Three-dimensional sediment trans- port measurements by acoustics (tridisma). Proc. Oceans conf., 2, 1108-1114.

[69] Terek B.2004. Comparison of river discharge measurements by conventional current meter and acoustic Doppler current profiler. BALWOIS 2004. Ohrid, Macedonia.

[70] Thorne P.D. \& HANes D.M. 2002. A review of acoustic measurement of small-scale sediment processes. Continental Shelf Research, 22 (4), 603-632.

[71] Védie F.2005. Régimes d'écoulements aux embouchures de bras morts. Etude hydraulique de la lône de Port-Galland. Lyon, Cemagref. Rapport de stage ENGEES n ${ }^{\circ}$ CHHLY.17134.

[72] Villard P., Church M.A. \& Kostaschuk R. 2005. Estimating bedload in sand-bed channels using bottom tracking from an acoustic Doppler profiler. Spec. Publs int. Ass. Sediment, 35, 197-209.

[73] Wang F. \& Huang H.2005. Horizontal Acoustic Doppler Current Profiler (H-ADCP) for real-time open channel flow measurement : flow calculation model and field validation. XXXI IAHR congress. Seoul, Korea.

[74] Wren D.G., Barkdoll B.D., Kuhnle R.A. \& Derrow R.W.2000. Field techniques for suspended-sediment measurement. Journal of Hydraulic Engineering, 126 (2), 97-104.

[75] Yorke T.H. \& OBerg K.A.2002. Measuring river velocity and discharge with acoustic Doppler profilers. Flow Measurement and Instrumentation, 13, 191-195. 